\title{
The ratio of viscosity to entropy density in a pion gas satisfies the KSS holographic bound
}

\author{
A. Dobado, F.J. Llanes-Estrada ${ }^{a}$ \\ Departamento de Física Teórica I, Universidad Complutense, 28040 Madrid, Spain
}

Received: 25 September 2006 / Revised version: 30 October 2006 /

Published online: 11 January 2007 - (c) Springer-Verlag / Società Italiana di Fisica 2007

\begin{abstract}
We evaluate the ratio of shear viscosity to entropy density in a pion gas employing the UehlingUehlenbeck equation and experimental phase-shifts parameterized by means of the $S U(2)$ inverse amplitude method. We find that the ratio for this monocomponent gas stays well above the KSS $1 /(4 \pi)$ bound. We find similar results with other sets of phase-shifts and conclude that the bound is nowhere violated.
\end{abstract}

PACS. 05.20.Dd; 51.20.+d

Recently, Kovtun, Son and Starinets [1] have conjectured a universal bound for the viscosity to entropy ratio of any one-component dilute gas based on gravity duality arguments and the Heisenberg uncertainty principle. In natural units the conjecture is that the quotient of the shear viscosity to the entropy density $\eta / s$ is greater than or equal to $4 \pi$, for any quantum field theory with one field alone.

A general proof does not exist but no counterexample is yet known either. This has caused considerable stir in the heavy-ion collision community as RHIC experiments are providing us with a picture of strongly interacting matter [2] that is close to a perfect fluid and features low viscosity.

Prospects for an indirect measurement of the viscosity in heavy-ion collisions through sufficiently precise hydrodynamic codes remain appealing [3].

Follow-up studies are under preparation from the hadron and from the quark-gluon phases in RHIC theory to see the effect of the phase transition on the viscosity.

We have presented a comprehensive study of the shear viscosity of a hadronic gas at low and moderate temperature [4]. Other transport coefficients are under active study; for example, there is a recent paper featuring the electrical conductivity of the pion gas in chiral perturbation theory [5] and a recalculation of the thermal conductivity is under preparation. In this brief report we employ our published results to address the viscosity to entropy density ratio.

We employ the same notation and conventions as in our earlier publication [4]. In particular we employ the $S U(2)$ inverse amplitude method parametrization of the pion-pion scattering experimental phase-shifts (as well as

\footnotetext{
a e-mail: fllanes@fis.ucm.es
}

alternative parametrizations to check the sensitivity of the calculation presented).

To show a consistent ratio where both numerator and denominator, $\eta$ and $s=S / V$, are computed with the same approximation, we need to realize that the viscosity of the pion gas is defined hydrodynamically near equilibrium; that is, for infinitesimally small gradients of the gas velocity field. Therefore the same approximation can be taken in the calculation of the entropy, and this has to be evaluated at equilibrium employing the Bose-Einstein distribution function $f_{0}$.

Further, the Uehling-Uehlenbeck (quantum Boltzmann) equation is decoupled from the BGKY hierarchy by assuming low density; that is, between two consecutive interactions, well separated by a large mean free path, particles decorrelate and behave as if the density of available states corresponded to a free gas. Therefore it is also fair to employ the free gas approximation in the computation of the entropy density.

Hence we are entitled to write

$$
\log Z=-V g \int \frac{\mathrm{d} \mathbf{p}}{(2 \pi)^{3}} \log \left[1-\mathrm{e}^{-\left(E-\mu_{\pi}\right) / T}\right] .
$$

Note that the chemical potential changes the density function $n(T)$

$$
n(y, z)=\frac{g_{\pi} m_{\pi}^{3}}{4 \pi^{2}} \int_{0}^{\infty} \mathrm{d} x \frac{x^{1 / 2}}{z^{-1} \mathrm{e}^{y(\sqrt{1+x}-1)}-1}
$$

in terms of the fugacity $z=\mathrm{e}^{\left(\mu_{\pi}-m_{\pi}\right) / T}$ and with $y=\frac{m_{\pi}}{T}$.

The total entropy follows from

$$
S=\frac{\partial(T \log Z)}{\partial T}
$$


and the entropy density, after dividing by the volume and integrating once by parts, becomes

$$
s=\frac{g}{6 \pi^{2} T^{2}} \int_{0}^{\infty} p^{4} \mathrm{~d} p \frac{\mathrm{e}^{\left(E-\mu_{\pi}\right) / T}}{\left(\mathrm{e}^{\left(E-\mu_{\pi}\right) / T}-1\right)^{2}} .
$$

In Fig. 1 we show the quotient of our previous computation of the viscosity divided by the entropy density for various temperatures and fugacities. It is clear that the holographic bound is at all times respected. Note that while the original paper [1] had zero chemical potential, the recent $N=4$ super-Yang-Mills computation in [6] shows that the bound is still valid for arbitrary $\mu$. This is indeed the case for the pion gas as we find.

To check the dependence of this quotient on the interaction chosen, we plot in Fig. 2 the same $\eta / s$ but the viscosity has been evaluated not only with the inverse amplitude method (IAM) [7], but also with another reasonable parametrization of the scattering phase-shifts [8], also with a constant cross-section based on Weinberg's scattering lengths:

$$
\sigma=\frac{23}{384} \frac{m_{\pi}^{2}}{\pi f_{\pi}^{4}}
$$

and finally with the full leading order in chiral perturbation theory

$$
|T|^{2}=\frac{1}{9 f_{\pi}^{4}}\left(21 m_{\pi}^{4}+9 s^{2}-24 m_{\pi}^{2} s+3(t-u)^{2}\right) .
$$

Employing the latter LO result, the authors of [9] find a cross-over violating the $\frac{1}{4 \pi}$ bound. We can discuss this by

\section{$\eta / s$}

Pion gas with IAM phase shifts for the viscosity, free entropy

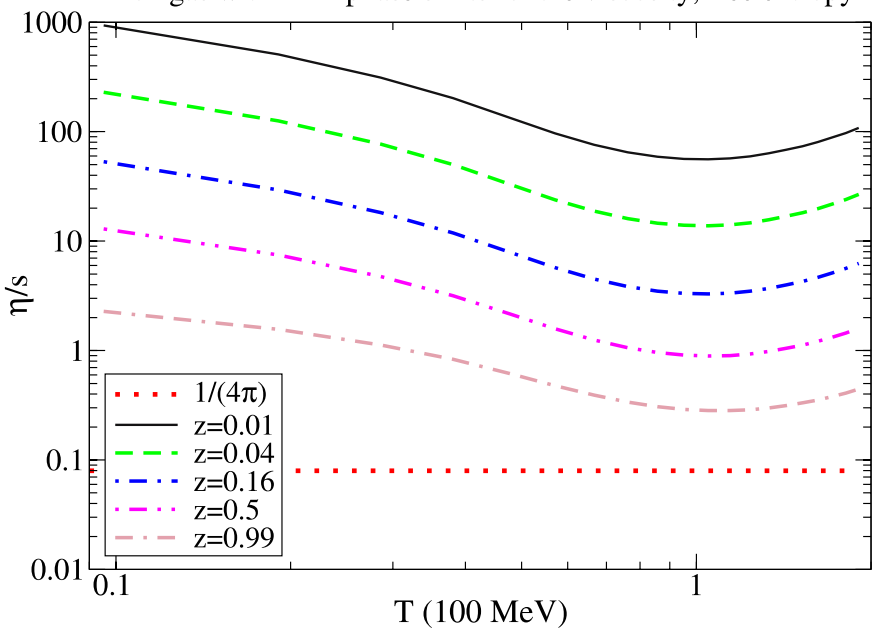

Fig. 1. Viscosity over entropy density as a function of the temperature for various chemical potentials (fugacities $z=$ $\left.\mathrm{e}^{\left(\mu_{\pi}-m_{\pi}\right) / T}\right)$. We employ the IAM parametrization of the pion-pion scattering phase shifts. As can be seen, the bound of Kovtun, Son and Starinets is respected and no hint of a crossing or phase transition appears from $\eta / s$ within this monocomponent pion gas $\eta / \mathrm{s}$ at $\mathrm{z}=0.01$

Pion gas, various phase shifts for the viscosity, free entropy

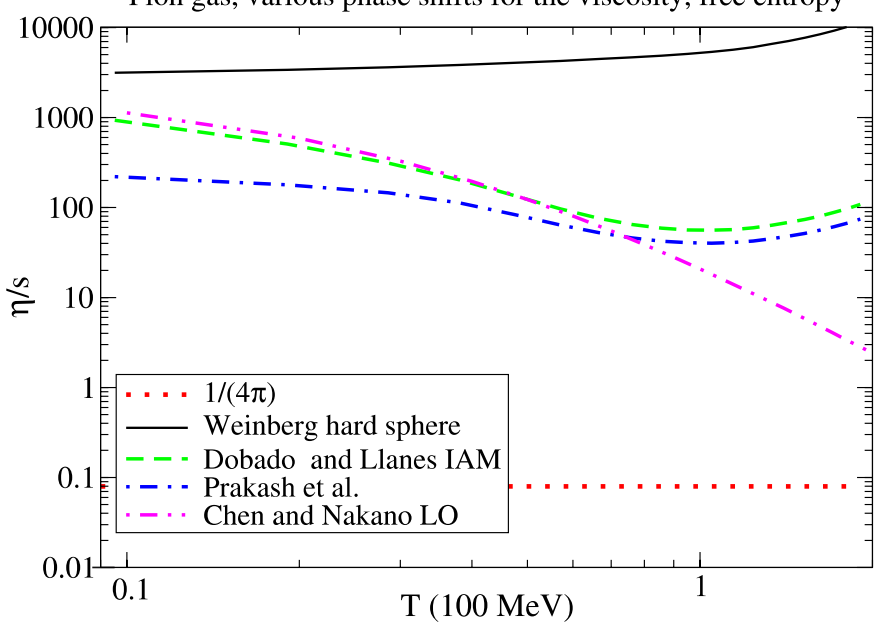

Fig. 2. Viscosity over entropy density as a function of the temperature for fixed fugacity $z=0.01$. We show various choices of interactions. The solid line at the top employs Weinberg's scattering lengths and the scattering amplitude is constant. The similar results of the IAM and the simple fit of Prakash et al. [8] for the scattering phase-shifts, naturally varying with momentum, mark what the ratio should be in the pion gas at moderate temperatures of order 50-100 MeV. Finally, the LO result in chiral perturbation theory of [9], growing with the average momentum in a collision indefinitely as a polynomial, greatly diminishes the viscosity and therefore its quotient by the entropy density

allowing the chemical potential to approach $m_{\pi}$ by choosing $z=0.99$, the results being plotted in Fig. 3. There we see that this crossing is an artifact of the leading order

$\eta / \mathrm{s}$ at $\mathrm{z}=0.99$

Pion gas, various phase shifts for the viscosity, free entropy

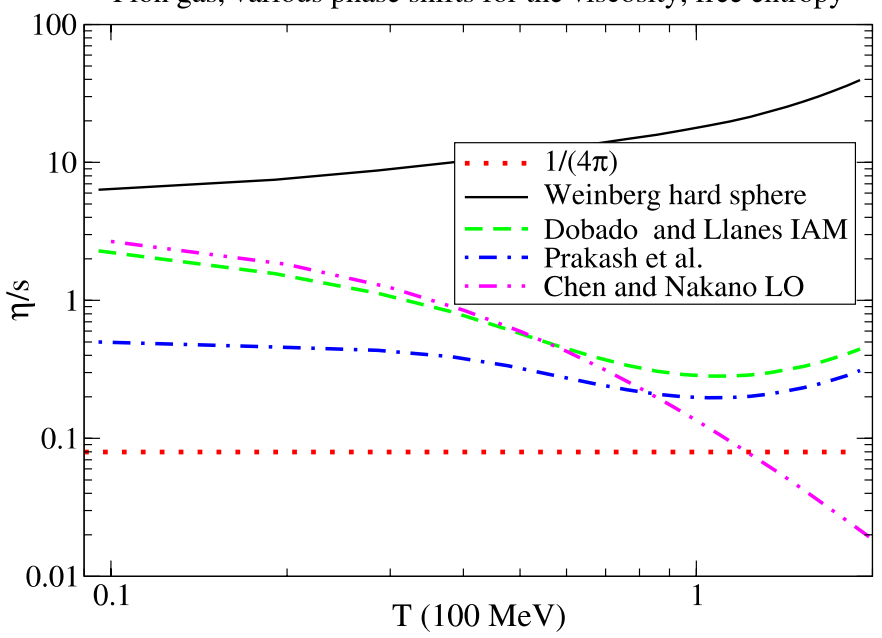

Fig. 3. Same as in Fig. 2. The ratio computed in LO- $\chi \mathrm{PT}[9]$, growing with the average momentum in a collision indefinitely as a polynomial, has an unphysical cross-over below the Kovtun-Son-Starinets bound that is not supported by realistic phase-shifts 
$\eta / \mathrm{s}$

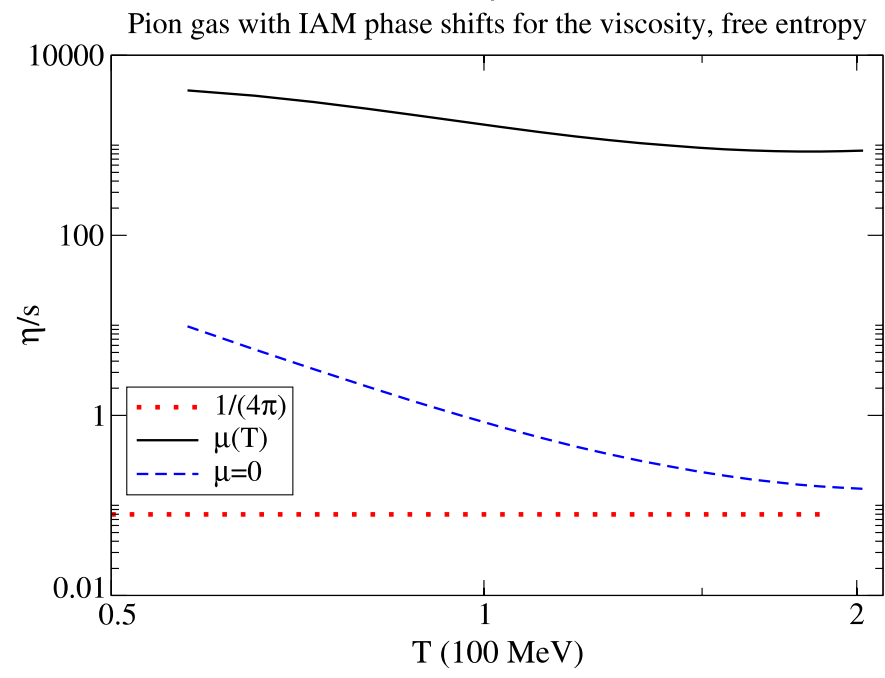

Fig. 4. Same as in Fig. 2 except we do not employ fixed fugacity. We plot the quotient $\eta / s$ as function of temperature for $\mu=0$ (for comparison) and for $\mu$ given in (7), that is a rough guess of its evolution in a heavy-ion collision. Reading this graph from right to left we can see that the quotient increases with the expansion of the pion gas. The possibility of a minimum at a phase transition is not discarded and further work in this direction is necessary including other mesons, as approaching the critical temperature populates successively other flavors and spins (and eventually nucleons)

chiral perturbation theory and is not to be expected from any realistic parametrization of the scattering phase-shifts, cutting all speculation about a phase transition being visible from $\eta / s$ in this one-component gas. The reason is simple: unitarity at the $\rho$ pole in pion-pion scattering tames the amplitude growth that is unchecked in LO- $\chi \mathrm{PT}$. This unphysical growth reflects in an ever-decreasing viscosity, sending $\eta / s$ to zero and violating any bounds. The IAM correctly incorporates elastic unitarity and is free from this feature. The viscosity computed with this method is moreover comparable with published works [10]. Even at the highest fugacity, $\eta / s$ is a factor of 2 above the $\frac{1}{4 \pi}$ bound.

After ensuring that this bound is nowhere violated, one wonders whether the observation [2] of a minimum of $\eta / s$ might be a useful signature of a phase transition in heavyion collisions. To address this we need a model for the evo- lution of the pion chemical potential with the temperature. Then one can think of the temperature as a clock ticking the time after the phase transition in reverse. We can take the following model from [11], consistent with the thermal photon spectrum in such collisions:

$$
\mu_{\pi}=\frac{2-T}{1.05}
$$

(both $T$ and $\mu$ expressed in units of $100 \mathrm{MeV}$ ). The result is plotted in Fig. 4. For comparison we also plot the fixed $\mu=0$ case. As can be seen, the viscosity decreases smoothly and the possibility of a minimum at the phase transition is not ruled out by the present analysis. To make this conclusion stronger one would need to populate further resonances in the hadron gas. This work will be undertaken in the near future.

Acknowledgements. We thank Jochen Wambach for encouraging us to carry out this interesting calculation, Juan M. Torres Rincón for useful comments, and Angel Gomez Nicola, Daniel Fernandez Fraile and Dany Davesne for feedback on their own evaluation of transport coefficients in a pion gas. This work has been supported by grants FPA 2004-02602, 2005-02327, PR27/05-13955-BSCH (Spain).

\section{References}

1. P. Kovtun, D.T. Son, A.O. Starinets, Phys. Rev. Lett. 94, 111601 (2005) [hep-th/0405231]

2. L.P. Csernai, J.I. Kapusta, L.D. McLerran, Phys. Rev. Lett. 97, 152303 (2006) [nucl-th/0604032]

3. D. Teaney, Phys. Rev. C 68, 034913 (2003) [nucl-th/ 0301099]

4. A. Dobado, F.J. Llanes-Estrada, Phys. Rev. D 69, 116004 (2004) [hep-ph/0309324]

5. D. Fernandez-Fraile, A. Gomez Nicola, Phys. Rev. D 73, 045025 (2006) [hep-ph/0512283]

6. D.T. Son, A.O. Starinets, JHEP 0603, 052 (2006) [hepth/0601157]

7. A. Dobado, J.R. Pelaez, Phys. Rev. D 56, 3057 (1997) [hep$\mathrm{ph} / 9604416]$

8. M. Prakash, M. Prakash, R. Venugopalan, G. Welke, Phys. Rep. 227, 321 (1993)

9. J.W. Chen, E. Nakano, hep-ph/0604138

10. D. Davesne, Phys. Rev. C 53, 3069 (1996)

11. W. Liu, R. Rapp, nucl-th/0604031 